\title{
BMJ Open Use of technology in supporting goal setting in rehabilitation for adults: a scoping review
}

\author{
Carla Strubbia (D) , ${ }^{1}$ William Mark Magnus Levack (D) , ${ }^{1}$ Rebecca Grainger (D) ,2 \\ Kayoko Takahashi, ${ }^{3}$ Kounosuke Tomori ${ }^{4}$
}

To cite: Strubbia C, Levack WMM, Grainger R, et al. Use of technology in supporting goal setting in rehabilitation for adults: a scoping review. BMJ Open 2020;10:e041730. doi:10.1136/ bmjopen-2020-041730

- Prepublication history and supplemental material for this paper are available online. To view these files, please visit the journal online (http://dx.doi. org/10.1136/bmjopen-2020041730).

Received 16 June 2020

Revised 25 August 2020

Accepted 23 September 2020

Check for updates

(c) Author(s) (or their employer(s)) 2020. Re-use permitted under CC BY-NC. No commercial re-use. See rights and permissions. Published by BMJ.

${ }^{1}$ Department of Medicine, University of Otago, Wellington, New Zealand

${ }^{2}$ University of Otago, Wellington, New Zealand

${ }^{3}$ Department of Occupational Therapy, Kitasato University, Sagamihara, Kanagawa, Japan ${ }^{4}$ Department of Occupational Therapy, Tokyo University of Technology, Ohta-ku, Tokyo, Japan

Correspondence to Carla Strubbia; carla.strubbia@postgrad.otago. ac.nz

\section{ABSTRACT}

Objective To map the extant literature evaluating the use of technology for goal setting in adult rehabilitation and the impact of technology for patient outcomes.

Design Scoping review.

Methods MEDLINE (via Ovid), CINAHL (via EBSCO),

AMED and Scopus were searched for articles describing observational or interventional studies. ProQuest

Dissertations and Theses database were searched for grey literature. Two review authors independently screened all titles and abstracts for potentially relevant articles. We included articles describing studies that had evaluated the development or application of technology to facilitate goal setting in rehabilitation for adults. Articles were excluded if the technology described did not include features to facilitate goal setting or were not in English. Narrative reviews, opinion pieces and editorials were also excluded. Results After screening 1640 publications of potential interest, we identified 27 studies for inclusion. These 27 articles described studies involving a total of 16 different technologies including, seven mobile apps, three websites, two mobile apps/website hybrids, two apps and two websites connected to a pedometer. We found that most technologies described were designed to facilitate selfmanagement with goal setting as a feature and that only five included a shared decision moment around goal setting. Only six of the 16 technologies had research providing evidence of effectiveness in terms of improved patient outcomes, with the best evidence of beneficial effects associated with technologies that linked goal setting to pedometer use.

Conclusions The identified technologies for use in adult rehabilitation that included goal setting as a feature were largely accepted and valued by patients and health professionals. The limited data suggest that there is a need for further research; specific foci may include the impact of incorporation of a shared decision-making moment and evaluation of effectiveness on patient outcomes.

\section{INTRODUCTION}

Goal setting is an essential component of any rehabilitation programme. This concept has increasingly dominated discourse as the formal process whereby a rehabilitation professional or a multidisciplinary team, together with the patient and/or their family, negotiate goals. Goal setting has been

\section{Strengths and limitations of this study}

This scoping review synthesises research evidence in a relatively new research area and identifies areas for further research.

- Knowing that digital technologies rapidly change, the reported findings may need to be reappraised in the near future.

- The nature of the scoping review did not allow the grading of the evidence since a quality evaluation of the included studies has not been conducted.

described as a desired future state that has to be actively selected, intentionally created, have purpose and is shared ${ }^{1}$ and is an essential feature of any rehabilitation programme. ${ }^{23}$

Goal setting in rehabilitation ensures explicit identification of the reasons for any clinical activity. In rehabilitation, setting goals may: increase patient motivation and drive; allow the rehabilitation process to be better monitored and so to stop ineffective interventions; enhance the effectiveness of therapy and enhance shared decision making and thus the person-centredness of rehabilitation service delivery. ${ }^{45}$ Locke and Latham stated that goals have an energising function whereby participation in the goalsetting process increases performance, and persistence to achieve specific goals. ${ }^{6}$ As well, Wade affirmed the identification and setting of appropriate collaborative goals should be one of the skills that characterises professionals involved in rehabilitation. ${ }^{7}$

Despite the importance attributed to goal setting, health professionals can struggle to involve patients in the goal selection process to the extent that is desirable. ${ }^{8-11}$ This lack of involvement may be due to: (a) patients being hesitant to promote their own ideas as they rely on the health professional to direct rehabilitation planning ${ }^{12}$; (b) health professionals being overwhelmed by the task of collaborating in goal setting and with goal setting 
interactions with other professionals, patients and families being perceived as complex, difficult and requiring effort $^{13}$; (c) lack of clinician education on how to engage patients in goal setting or (d) lack of patient interest in participating. ${ }^{14}$

The use of technologies such as websites, smartphones and apps that generate, store or process data has been suggested as increasing engagement of patients in goalsetting. ${ }^{14-16}$ In particular, mobile apps and websites may enable health professionals to more adequately engage patients and their families in goal setting. Technologies could enable goal setting by: (a) providing the shared space where patients can offer their ideas and opinions about their goals; (b) providing a structure for collaboration in goal setting, while also reducing or managing complexity in goal setting; (c) overcoming the lack of clinician education by intentional design to engage patients in goal setting or (d) increasing patient interest in goal setting through appealing design and easy-to-use features. A recent study evaluating a mobile app designed to target impairments and improve quality of life for people with schizophrenia found participants actively engaged with the mobile app each time they logged in. Moreover, participant's satisfaction levels were high, particularly regarding the features associated with setting personalise goals. ${ }^{17}$ Another study assessing a web-based intervention designed to enhance self-efficacy to selfmanage cancer-related fatigue after primary cancer treatment, showed that participants engaged well with the goal-setting sections of the website. ${ }^{18} 19$ Despite these findings, most studies exploring technology for goal setting have highlighted the need to gain further knowledge and empirical data regarding impact, and that refinements to health technologies are required. ${ }^{20-22}$ This study aimed to map the extant literature that evaluates the use of technology for goal setting in adult rehabilitation and the impacts of technology for patient outcomes.

\section{METHODS}

\section{Research design}

The purpose of a scoping review is to comprehensively synthesise evidence to map a broad, complex or emerging field of study and to identify gaps with the intent to inform practice, policy and future research. ${ }^{23}$ We chose to use the scoping review methodology to broadly explore and summarise the literature about the use of technology around goal setting in rehabilitation. We used the framework recommended by Arksey and O'Malley, ${ }^{23}$ further developed by Levac et $a l^{24}$ and finally enhanced by the work of Peters et al. ${ }^{25}$ This five-step process comprised: (a) identifying the research question, (b) identifying relevant studies, (c) selecting studies, (d) charting data and (e) collating, summarising and reporting results. The protocol for this review was prepublished online. ${ }^{26}$ This review was reported using the Preferred Reporting Items for Systematic Reviews and Meta-Analyses-Extension for scoping reviews (PRISMA-ScR). ${ }^{27}$ The completed
PRISMA-ScR checklist is provided in online supplemental appendix 1.

\section{Identification of the research objectives}

The research objectives were identified from a preliminary scan of the literature and from the expertise of the research team. The research team includes a physiotherapist (CS), an academic physiotherapist (WMML) with expertise in goal setting, an academic physician with interests in technology (RG), and two academic occupational therapists (KTakahashi, KTomori). Moreover, given the iterative nature of the scoping review that allows the researcher to reproduce, manipulate and re-analyse data to add to the results to support the interpretation of the study, we adapted and reframed the objectives of the study with the data extraction process. Rationale for the questions arose from the lack of studies in the academic literature on the use of the digital technologies in the goal setting process in rehabilitation settings.

The primary objective of this scoping review was to identify and summarise the research that has examined the application of technology for goal setting in rehabilitation. A secondary objective was to gather and synthesise research on the effectiveness of technology for goal setting in rehabilitation to improve patient outcomes.

\section{Identification of relevant articles, inclusion and exclusion criteria}

Types of studies

We included studies published at any time in English that explored the development or application of information and communication technology (internet-based technology and mobile apps) to facilitate goal setting in rehabilitation. Methodological quality of the published articles was not a criterion for exclusion/inclusion, so that a breadth of knowledge pertaining to the research objectives could be included, as is consistent with scoping review practices. ${ }^{23} 2428$ Review publications, experimental trials (eg, randomised controlled trials (RCTs)), observational studies (eg, cohort studies), case studies and qualitative studies were included. We excluded narrative reviews, opinion pieces and editorials.

\section{Type of participants}

Studies involving participants aged 18 years or over, with any health conditions, as defined by the International Classification of Disease ${ }^{5}$ who were receiving rehabilitation were included. We used the conceptual description provided by Meyer $e t a l^{29}$ to determine what 'rehabilitation' was:

Rehabilitation is a health strategy which, based on the World Health Organization's integrative model of functioning, disability, and health, applies and integrates [assessments and therapeutic interventions]... in partnership between person and provider... to enable persons with health conditions experiencing or likely to experience disability to achieve and maintain optimal functioning (p768). ${ }^{29}$ 


\section{Types of technology}

We included studies that described or evaluated goal setting technology that had been designed specifically for rehabilitation services as well as goal setting technology designed for general use but applied to rehabilitation services. This included computer programmes, websites and mobile apps (eg, apps for smartphones and tablets) designed to help people set rehabilitation goals. We included technology that was used to: (a) facilitate the selection of a topic for goals to be set around, including negotiation of this topic between patients, family and health professionals; (b) help select a specific target (ie, level of performance) to be achieved by a patient at a point in the future; (c) facilitate the translation of a topic and specific target for a goal into an explicit goal statement (ie, writing and recording the goal statement); and (d) renegotiate the topic or targeted level of performance for a goal.

We excluded studies that investigated the development or application of technology primarily designed to aid communication (eg, augmented and alternative communication or telemedicine technology), without being specifically designed for goal setting. We also excluded studies that investigated the development or application of technology that was only used for: (a) collecting assessment (ie, baseline) data; (b) providing information to patients (eg, about health conditions or the rehabilitation process); (c) reporting or measuring performance data, even if this was against stated goals (eg, pedometers to measure step count against a goal related to walking where walking was a general activity rather than a specific rehabilitation goal were excluded) and/or (d) sending motivational messages or reminders to patients, without the technology also being used to aid the setting of goals.

\section{Search strategy}

A search strategy for Medline (via Ovid), CINAHL (via EBSCO), AMED, Scopus and ProQuest were developed in collaboration with a reference librarian. Searching was last executed the 20 May 2020. The search strategy for all databases is in online supplemental appendix 2 . Reference lists and forward citation searches of included studies (using Scopus and Google scholar) were also searched and any potentially relevant studies included.

\section{Data screening}

We imported identified articles into the reference manager Endnote (V.X9, Clarivate Analytics), then uploaded them to Rayyan QCRI web application, ${ }^{30}$ where the titles and abstracts were reviewed to eliminate duplicates. Two authors (CS, WMML) separately screened all article titles and abstracts for inclusion against eligibility criteria. The full text of all eligible articles then underwent independent review by two authors (CS, WMML) to confirm if inclusion criteria were met. At each stage, any discrepancies were discussed until consensus was reached, with third author (KTo) adjudication as needed. The discrepancies found were about the goal setting features of the technology, the population, and the design of the study as well as the authors' inaccuracy in reading the title and abstract. Two authors (CS, WMML) reached consensus by reviewing the full-text article, downloading and using the app/website to check if was designed for goal setting and consulting a third author. All discrepancies were easily resolved and did not require further action. Twentythree discrepancies were found, and only two resulted in consulting a third author (KTo). One record was eliminated because was a protocol and the second because the goal setting component of the technology was not clearly stated. Where articles included study authors who were also on the review team, those authors were excluded from decisions about or data extraction from that article.

Charting, summarising and reporting the results of the review A descriptive-analytical narrative method was used to extract and chart the data. ${ }^{2324} 28$ The first author (CS) drafted the data extraction template, which was further refined after feedback from all authors. Five articles were used to pilot the data extraction tool. Two authors independently extracted the data for each article. The primary author (CS) then extracted data from all articles, with the other authors each extracting data from a quarter of the included articles. All discrepancies were reviewed in discussion between the authors (CS and WMML for all, with RG, KTakahashi or KTomori) and agreed by consensus. The final version of the data extraction form is in the online supplemental appendix 3 . We extracted the data on characteristics of each included article and the key study findings. For key study findings, we collected quantitative and qualitative data on intervention effectiveness and user perceptions of the technology. Given the heterogeneity of the included studies, no secondary analysis of published data (eg, meta-analysis or metasynthesis) was undertaken. The primary author (CS) used an Excel spreadsheet to collate and analyse extracted data. A risk of bias assessment of the included studies was not performed as this is outside of the objectives of a scoping review. ${ }^{27}$

\section{Patient and public involvement}

No patients or members of the public were involved.

\section{RESULTS}

\section{Search strategy}

Our search identified 1071 unique records, after removal of duplicates. We excluded 1013 records on screening titles and abstract, and a further 36 on full-text screening, leaving 22 articles for data charting from the database searches (see figure 1). Reasons for excluding the 1031 records at titles and abstracts included: absence of the word 'goal setting' in the abstract; technology not used in a rehabilitation context; non-adult population and narrative reviews, editorials and opinion pieces. Reasons for excluding the 58 articles at full-text screening included: insufficient information about the goal setting process 


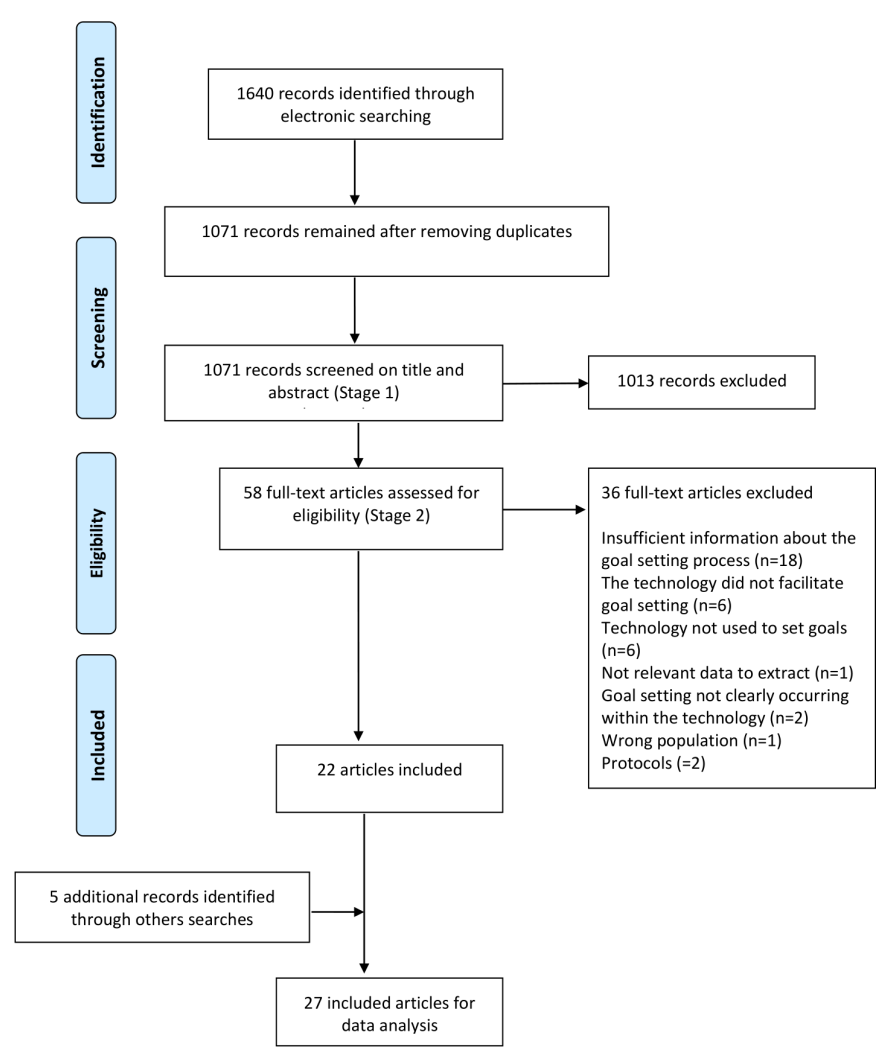

Figure 1 Preferred Reporting Items for Systematic Reviews and Meta-Analyses-Extension for scoping reviews flow diagram for article selection.

$(\mathrm{n}=18)$; technology did not facilitate goal setting process $(\mathrm{n}=6)$; technology was not used to set goals $(\mathrm{n}=6)$; lack of relevant data to extract $(n=1)$; goal setting did not clearly occur within the technology $(n=2)$; wrong population $(\mathrm{n}=1)$; and the article was a study protocol $(\mathrm{n}=2)$. Five more articles were identified through checking reference lists and forward citation searching, giving a total of 27 articles for data extraction.

\section{Characteristics of included studies}

Most of the articles $(22 / 27,81.5 \%)$ were published in the last 5 years. The studies were from the USA $(10 / 27$, $37.1 \%)$, UK $(5 / 27,18.5 \%)$, Canada $(3 / 27,11.1 \%)$, Japan $(3 / 27,11.1 \%)$, an international partnership between Finland, Sweden, Texas and India $(1 / 27,3.7 \%)$, an international partnership between New Zealand, Australia, UK and USA $(1 / 27,3.7 \%)$ a European partnership between Belgium, Greece, UK, Switzerland and Netherlands $(1 / 27,3.7 \%)$, Korea (1/27, $3.7 \%)$, New Zealand (1/27, $3.7 \%$ ) and Portugal $(1 / 27,3.7 \%)$. Common study designs were RCTs in which the technologies were evaluated $(8 / 27,29.6 \%)$ and observational studies $(7 / 27,25.9 \%)$, with smaller numbers of qualitative studies exploring the stakeholders views about the technologies for goal settings $(5 / 27,18.5 \%)$, mixed-methods studies (4/27, $14.8 \%$ ), descriptive studies eliciting opinions concerning digital technologies around goal settings $(2 / 27,7.5 \%)$ and a pilot study $(1 / 27,3.7 \%)$.

\section{Characteristics of included technologies}

Among the 27 articles, there were 16 different programmes/software described, which were 7 mobile apps, 3 websites, 2 mobile app/website hybrids (the mobile app was connected with a corresponding website) and technologies (2 mobile apps and 2 websites) connected to a pedometer. Most of the 16 technologies were diseasespecific $(12 / 16,75 \%)$ designed for a named condition: heart conditions $(n=3)$, neurological disorders $(n=2)$, diabetes $(n=2)$, chronic obstructive pulmonary disease (COPD) $(\mathrm{n}=2)$, schizophrenia $(\mathrm{n}=2)$ and cancer-related fatigue $(n=1)$. The remaining technologies $(4 / 16,25 \%)$ were for adults with any chronic condition or multiple chronic conditions. Almost half of the included technologies $(7 / 16,43.8 \%)$ were, at the time of the data charting, available in the market for public purchase from the Google Play store, Apple's app store, Microsoft's app store or from a website. The remainder $(9 / 16,56.3 \%)$ were not accessible: four were under development, three were associated with a private health service, one had a region/location restriction and one for no identifiable reason. By downloading the app or accessing the website, reading the app store description and scanning the information in the article, we found that the majority of technologies described $(10 / 16,62.5 \%)$ were designed for self-management use and that the remaining six $(6 / 16$, $37.5 \%$ ) were also for self-management, but with the possibility of having feedback or evaluation from a health professional.

\section{Characteristics of approaches to goal setting}

For most of the technologies $(9 / 16,56.3 \%)$, goal selection was undertaken by the patient. The remaining technologies included: two that involved automated goal setting with no direct human control, setting treatment objectives from a predetermined list $(2 / 16,12.5 \%)$, and five that allowed a shared decision moment, in which client and health professional could meet in person or online to decide on the common objectives $(5 / 16,31.3 \%)$. Most of the technologies $(11 / 16,68.8 \%)$ facilitated the evaluation of goal achievement and provided a report of each client's goal progress, represented by bar graphs, illustrations or figures. For 9 of the 16 technologies (56.3\%), there was no identified psychological theory underpinning the approach to goal setting. For the remaining 7 of the 16 technologies $(43.7 \%)$ the psychological theories used included: Bandura's Social Learning Theory, ${ }^{18} 193132$ Self-Determination Theory, ${ }^{14} 1733$ Behavioural Change Theory ${ }^{34-37}$ and Problem Solving Theory. ${ }^{38}$

\section{Evidence of the user experience}

Overall patients reported positive experiences of the technologies included, especially how easy to use or pleasing the technology was. The technology needed to be straightforward and easy to operate, with clear and simple instructions and good accessibility, including for those with lower levels of digital literacy. ${ }^{31} 39$ One study showed that people less familiar with the internet would 
need support, and that individuals with a preference for written media were more likely to drop out of studies. ${ }^{19}$ Another study indicated that older individuals had more difficulties using these technologies. ${ }^{37}$ Users wanted their technology to be able to track goals, physical activity and other health measures, such as heart rate, weight and diet, ${ }^{370}$ as well as have more specific and individualised relevant goals and personalised feedback from health professionals. ${ }^{31}{ }^{41}$ Devi et al conducted semistructured interviews with 16 participants to explore patient experiences of using a web-based cardiac rehabilitation programme. These participants increased physical activity, improved psychological well-being and demonstrated greater empowerment when managing angina symptoms; the programme also instilled confidence related to exercise. ${ }^{34}$ One study showed that most of the participants were able to identify beneficial outcomes from taking part on the study, and that the technology helped them to gain a better understanding of their disease and its management. Participants liked interactive technology and video links to patient stories, which were perceived to be more engaging than paper-based information. ${ }^{31}$

\section{Evidence regarding the health effects of the included technologies}

Among the included articles, eight were RCTs assessing the effectiveness of six different technologies. These studies evaluated technologies including websites only $(\mathrm{n}=2),{ }^{18} 32$ websites synchronised to a pedometer $(n=4),{ }^{35} 42-44$ a mobile app $(n=1)^{17}$, and a mobile app linked to a pedometer $(\mathrm{n}=1) .{ }^{45}$ Primary outcome measures for the studies included daily step count ( $\mathrm{n}=5$ studies with technology linked to pedometer) and usage and adherence to the technology $(n=3)$. Of note, goal setting was only one feature of many in all these technologies, so any benefits could not be attributed with the goal setting components alone. The studies with technology linked to a pedometer reported increases in daily step count in the intervention group compared to the control group, mostly in the first 4 months of use..$^{3542-45}$ However, in most of these studies there were no statistically significant between-group differences in any primary or secondary outcomes at any time points. Demeyer et $a t^{45}$ investigated the effectiveness of a 12-week semiautomated telecoaching intervention on physical activity in patients with COPD in a multicentre RCT. They showed that the app resulted in statistically significant improvements in step count and small changes in time doing moderate physical activity, but that the other outcomes including the overall health status and breathlessness, did not differ. Moy $e t a t^{42}{ }^{43}$ tested the efficacy of an internet-mediated, pedometer-based exercise intervention, again with a component of goal setting, in a population of US veterans with COPD. Differences in daily step counts in the intervention group compared with controls were statistically significant at 4 months, but not at 8 months.

Devi et $a l^{35}$ examined the effectiveness of a web-based cardiac rehabilitation programme for people with angina.
They randomised 95 people to either an intervention group using the website connected to an accelerometer, with goal setting around step count or a control group, who continued treatment as usual. The results showed that people in the intervention group obtained a statistically significant improvement in their goal of daily step count weight, and self-efficacy and a reduction in their duration of sedentary behaviour and angina frequency, both at 6 weeks and at 6 months follow-up.

The remaining studies showed that participants demonstrated large variability in website/app usage, especially for the first few months. Two of these studies reported no statistically significant between-group differences in any of the measured outcomes. Foster et al ${ }^{19}$ conducted an RCT where 85 people were allocated to either an intervention group, given access to a website that provided a goal-direct programme for self-management of cancerrelated fatigue, or to a control group, provided only with written information on fatigue management. The authors reported that there were no statistically significant differences in any of the scores at any time point. Similarly, Glasgow $e t a l^{32}$ conducted an RCT $(\mathrm{n}=270)$ to test the effectiveness of a website-based self-management intervention for people with type 2 diabetes mellitus, which involved a component of goal setting, in comparison to usual care. Participants demonstrated a large variability in website usage over the 4 month period decreasing over time and no significant differences between-groups on most of the engagement variables.

Only one small study, evaluating the efficacy of a mobilebased digital health intervention designed to improve motivation and quality of life in a 12-week RCT, demonstrated a significant increase in all outcomes measured. Compared with a group receiving usual care, the people in the intervention group had significantly greater improvements in self-reported depression, defeatist beliefs, self-efficacy and a trend towards motivation/pleasure negative symptoms post-trial, and these improvements were maintained 3 months after the end of trial. ${ }^{33}$ See online supplemental appendix 4 for more details.

\section{DISCUSSION}

This scoping review identified and summarised the literature that examines technology for goal setting in rehabilitation and the effectiveness of these technologies for improving patient outcomes. Our study showed that the 27 articles included 16 different technologies with the majority of those being mobile apps and the rest websites. Four of the technologies were linked to pedometers providing numeric feedback on progress towards goals. The purpose of goal setting in this context was to enhance physical activity by empowering participants to increase their walking as part of the rehabilitation process. ${ }^{42-45}$ Several studies have used step counters to provide realtime feedback on physical activity with goal setting, and coaching programmes, with the aim of increasing physical activity, have been associated with larger improvements 
in amount of physical activity compared with only rehabilitation. ${ }^{46}$

An interesting finding was that the majority of the technologies identified were meant to aid selfmanagement strategies, with the patients independently selecting their own goals. Fewer technologies allowed healthcare professionals or coaches, to have insight into patient adherence to a self-management programme or allow for additional intervention if patients were having difficulty with progressing towards a particular goal. For example, some technologies provided automated prompts to the patient to conduct self-management activities and allowed them to send information, including photographs, to clinicians to alert them of medical issues. ${ }^{14} 4045$ Others technologies allowed the contact with therapists in crisis situations. ${ }^{37}$ This finding aligned with evidence in the literature that technologybased interventions can help people manage physical symptoms ${ }^{47}$ and that interventions designed to increase self-efficacy to manage physical symptoms are effective in chronic-disease populations. ${ }^{48}$

Only a few of the included technologies were created with the intent of aiding therapists and patients to jointly set rehabilitation goals. ${ }^{1836394950}$ These technologies were developed following the recommendation that sharing decisions about goals can have a positive impact on the patient's health and mental well-being. ${ }^{51}$ Furthermore while a rich and sophisticated body of theory about goal setting in rehabilitation-with a sound empirical basealready exists ${ }^{52}$ less than half of the technologies examined were not based on any stated theoretical framework. Our data synthesis suggest that future goal setting technologies should be underpinned by best-practice principles of rehabilitation and evidence about goal setting.

The extent to which the scope or focus of goal setting was restricted in the technologies examined is also notable. Some technologies only permitted goals to be set on a limited set of topics, for example selecting from a menu of predetermined goals. Others only allowed goals to be set around level of performance in just one area of functioning, for example, step count goals. Only five technologies clearly allowed for an open-ended process of setting goals, thus enabling shared decision making and supporting person-centred approach to rehabilitation. 1431364153

Evidence shows that a move towards a person-centred, caring and strengths-based approach can promote engagement in rehabilitation. ${ }^{54}$ This process has been shown to facilitate disabled persons' voices to be heard, and their expertise and competence to be acknowledged, which may increase patient motivation and drive, achieving the first objective of goal setting. Therefore, person-centredness during technology development may lead to the improvement in intervention effectiveness/efficiency. ${ }^{55}$ Future technologies developed for goal setting in rehabilitation should also consider how the technology may specifically enhance the effectiveness of therapies and/or be useful for monitoring rehabilitation.
These objectives may require a wider focus on which types of goals can be included.

The eight RCTs that gave information about the effectiveness of the digital technologies for patient outcomes have shown that technologies that addressed the requirements of patients with complex care needs. Such patients tend to be high users of the healthcare system and may benefit from rehabilitation, ${ }^{56}$ and were identified as those who might benefit from a device that allows to set rehabilitation goals in the various aspects of their daily life. Results from these studies also showed that people enjoyed and positively accepted digital technologies as part or in place of their daily rehabilitation procedure. Several studies reported decreases in use after three or 4 months which may indicate familiarity with the technology and reduced novelty impact with ongoing engagement. Therefore, future research should investigate how to help people maintain engagement in goal-setting technologies over longer periods of time (eg, 6-12 months) or concentrate on benefits that may be valuable to achieve in short time periods.

\section{Limitations}

While this scoping review was planned, conducted and reported according to relevant guidelines ${ }^{27}$ various limitations need to be considered. One possible limitation of our study is the change in the research objectives from that stated in our archived protocol. Consistent with the iterative nature of the scoping review methodology, we adapted the objectives of the study in the initial stages of the data extraction process. Five articles we inlcuded were used to pilot the data extraction tool, with the tool then adapted to enable accurate data extraction from the other included studies (online supplemental appendix 3). After we clearly understood the types of studies described in the included articles, we adapted our research objectives; these steps are appropriate in a scoping review. ${ }^{25}$ Although this may be considered a limitation, the overall purpose of our scoping review remains unchanged, that is to comprehensively synthesise evidence and map the literature about the use of technology around goal setting in rehabilitation. A second limitation is about the quality assessment. Since scoping review methods do not require a quality assessment of included research papers, the study quality is likely to be variable. However, articles included were published in peer-reviewed journals, we excluded narrative reviews, opinion pieces and editorials and not all grey literature was included. Unpublished yet related information on the most current trends in this field may have been missed. Knowing that digital technologies rapidly change, the reported findings may need to be reappraised in the near future. This scoping review addressed a broad area of content and contexts, that is, different technologies, developers, features, goals and implementation contexts; multiple applications; different users, communities and countries and different multiple chronic conditions and single conditions. However, this may limit transferability of the results to a specific context 
and present as prime areas for future systematic and realist reviews.

\section{CONCLUSION AND IMPLICATION}

These results demonstrated that the web-based and mobile-based interventions were largely accepted and valued by the participants. We have also shown that there is the need for further research to develop technologies that sustain engagement and use over short periods and improve patient outcomes over the medium- to longterm. Researchers and developers of healthcare digital technologies may find benefits accrue when a theoretical framework is used to guide their cyclical, iterative process of design, implementation and evaluation. Clinical research could focus on developing more digital tools that are better integrated into clinical care in order to improve user acceptance, sustained engagement and ultimately, clinical value and behaviour change. These results have given an overall idea about how few technologies exists that specifically support a goal setting process that is collaborative, actively selected, patient-centred, shared and engaged in within the context of an interdisciplinary rehabilitation. Most of the technologies examined by this review were designed for very specific patient populations, and the goal setting components supported by a specific type of intervention. Many of the technologies placed significant restrictions on the type of goals that could be set and the topic. However, this review has also shown that there are in the market, even if still a minority, technologies that identify and facilitate shared discussion and prioritisation of meaningful, individualised goals, presenting patients with a wide variety of possible activities and social roles to provide an indication of the breadth of goals they could consider. The key to successful goal setting is supporting patients to become active partners in their health care. This should be accomplished by encouraging dialogue and questions, exploring values and stressors and celebrating successes. ${ }^{57}$ Effective goal setting requires active listening by providers as well as new approaches to patient counselling, and digital technologies can certainly support that. Additional research is critical in order to maximise the potential for digital health care, while advancing the triple aim of healthcare to improve patient's access, increase patient's satisfaction and, if possible, lower overall costs.

\section{Twitter Carla Strubbia @carlitwonder and Rebecca Grainger @drbeckyg}

Contributors CS conducted the study conception and design, and the analysis and interpretation of data, drafted the manuscript and revised content based on feedback. WMML and RG assisted with study conception and design, interpretation of data, and provided critical revision of drafts. KTa and KTo assisted with interpretation of data and provided critical revision of drafts. All authors approved the final version of the manuscript. CS is responsible for the integrity of this work as a whole.

Funding The authors have not declared a specific grant for this research from any funding agency in the public, commercial or not-for-profit sectors.

Competing interests None declared.

Patient consent for publication Not required.
Provenance and peer review Not commissioned; externally peer reviewed.

Data availability statement All data relevant to the study are included in the article or uploaded as supplemental information.

Supplemental material This content has been supplied by the author(s). It has not been vetted by BMJ Publishing Group Limited (BMJ) and may not have been peer-reviewed. Any opinions or recommendations discussed are solely those of the author(s) and are not endorsed by BMJ. BMJ disclaims all liability and responsibility arising from any reliance placed on the content. Where the content includes any translated material, BMJ does not warrant the accuracy and reliability of the translations (including but not limited to local regulations, clinical guidelines, terminology, drug names and drug dosages), and is not responsible for any error and/or omissions arising from translation and adaptation or otherwise.

Open access This is an open access article distributed in accordance with the Creative Commons Attribution Non Commercial (CC BY-NC 4.0) license, which permits others to distribute, remix, adapt, build upon this work non-commercially, and license their derivative works on different terms, provided the original work is properly cited, appropriate credit is given, any changes made indicated, and the use is non-commercial. See: http://creativecommons.org/licenses/by-nc/4.0/.

\section{ORCID iDs}

Carla Strubbia http://orcid.org/0000-0001-9324-3607

William Mark Magnus Levack http://orcid.org/0000-0001-6631-908X

Rebecca Grainger http://orcid.org/0000-0001-9201-8678

\section{REFERENCES}

1 Siegert RJ, Levack WMM. Rehabilitation goal setting: theory, practice and evidence. CRC press, 2014.

2 Barnes M, Ward AB. Textbook of rehabilitation medicine. Oxford, USA: University Press, 2000.

3 Cott CA. Client-centred rehabilitation: client perspectives. Disabil Rehabil 2004;26:1411-22.

4 Levack WM, Weatherall M, Hay-Smith JC, et al. Goal setting and strategies to enhance goal pursuit in adult rehabilitation: summary of a Cochrane systematic review and meta-analysis. Eur J Phys Rehabil Med 2016;52:400-16.

5 World Health Organization. WHO/CONRAD technical consultation on nonoxynol-9, world Health organization, Geneva, 9-10 October 2001: summary report. Reprod Health Matters 2002;10:175-81.

6 Locke EA, Latham GP. Building a practically useful theory of goal setting and task motivation. A 35-year odyssey. Am Psychol 2002;57:705-17.

7 Wade DT. Goal planning in stroke rehabilitation: how? Top Stroke Rehabil 1999;6:16-36.

8 Barnard RA, Cruice MN, Playford ED. Strategies used in the pursuit of achievability during goal setting in rehabilitation. Qual Health Res 2010;20:239-50.

9 Berg K, Askim T, Balandin S, et al. Experiences of participation in goal setting for people with stroke-induced aphasia in Norway. A qualitative study. Disabil Rehabil 2017;39:1122-30.

10 Levack WMM, Dean SG, Siegert RJ, et al. Navigating patientcentered goal setting in inpatient stroke rehabilitation: how clinicians control the process to meet perceived professional responsibilities. Patient Educ Couns 2011;85:206-13.

11 Rohde A, Townley-O'Neill K, Trendall K, et al. A comparison of client and therapist goals for people with aphasia: a qualitative exploratory study. Aphasiology 2012;26:1298-315.

12 Schoeb V, Staffoni L, Parry R, et al. "What do you expect from physiotherapy?": a detailed analysis of goal setting in physiotherapy. Disabil Rehabil 2014;36:1679-86.

13 Lloyd A, Roberts AR, Freeman JA. 'Finding a balance' in involving patients in goal setting early after stroke: a physiotherapy perspective. Physiother Res Int 2014;19:147-57.

14 Dicianno BE, Henderson G, Parmanto B. Design of mobile health tools to promote goal achievement in self-management tasks. JMIR Mhealth Uhealth 2017;5:e103.

15 Devi R, Singh SJ, Powell J, et al. Internet-based interventions for the secondary prevention of coronary heart disease. Cochrane Database Syst Rev 2015;12:CD009386.

16 Pyykkö I, Manchaiah V, Levo H, et al. Internet-based peer support for Ménière's disease: a summary of web-based data collection, impact evaluation, and user evaluation. Int J Audiol 2017;56:453-63.

17 Schlosser D, Campellone T, Kim D, et al. Feasibility of prime: a cognitive neuroscience-informed mobile APP intervention to enhance motivated behavior and improve quality of life in recent onset schizophrenia. JMIR Res Protoc 2016;5:e77. 
18 Foster C, Calman L, Grimmett C, et al. Managing fatigue after cancer treatment: development of restore, a web-based resource to support self-management. Psychooncology 2015;24:940-9.

19 Foster C, Grimmett C, May CM, et al. A web-based intervention (restore) to support self-management of cancer-related fatigue following primary cancer treatment: a multi-centre proof of concept randomised controlled trial. Support Care Cancer 2016;24:2445-53.

20 Levack W, Tomori K, Takahashi K, et al. Development of an Englishlanguage version of a Japanese iPad application to facilitate collaborative goal setting in rehabilitation: a Delphi study and field test. BMJ Open 2018;8:e018908.

21 Moon J, Heo SJ, Jung JH. A pilot investigation of Korean clients' occupation receiving rehabilitation using aid for decisionmaking in occupation choice. J Adv Res Dynamical Control Syst 2018;10:419-26.

22 Mortenson WB, Singh G, MacGillivray M, et al. Development of a self-management APP for people with spinal cord injury. J Med Syst 2019;43:145.

23 Arksey H, O'Malley L. Scoping studies: towards a methodological framework. Int J Soc Res Methodol 2005;8:19-32.

24 Levac D, Colquhoun H, O'Brien KK. Scoping studies: advancing the methodology. Implement Sci 2010;5:69.

25 Peters MDJ, Godfrey CM, Khalil H, et al. Guidance for conducting systematic scoping reviews. Int J Evid Based Healthc 2015;13:141-6.

26 Strubbia C, Tomori K, Grainger R. The use of technology in supporting goal setting in rehabilitation in adults: protocol for a scoping review, 2019.

27 Tricco AC, Lillie E, Zarin W, et al. PRISMA extension for scoping reviews (PRISMA-ScR): checklist and explanation. Ann Intern Med 2018;169:467-73.

28 Colquhoun $\mathrm{H}$, Leeman J, Michie S, et al. Towards a common terminology: a simplified framework of interventions to promote and integrate evidence into health practices, systems, and policies. Implement Sci 2014;9:781.

29 Meyer T, Gutenbrunner C, Bickenbach J, et al. Towards a conceptual description of rehabilitation as a health strategy. J Rehabil Med 2011;43:765-9.

30 Ouzzani M, Hammady H, Fedorowicz Z, et al. Rayyan-a web and mobile APP for systematic reviews. Syst Rev 2016;5:210.

31 Myall M, May CR, Grimmett C, et al. Restore: an exploratory trial of a web-based intervention to enhance self-management of cancerrelated fatigue: findings from a qualitative process evaluation. BMC Med Inform Decis Mak 2015;15:94.

32 Glasgow RE, Christiansen SM, Kurz D, et al. Engagement in a diabetes self-management website: usage patterns and generalizability of program use. J Med Internet Res 2011;13:e9.

33 Schlosser DA, Campellone TR, Truong B, et al. Efficacy of prime, a mobile APP intervention designed to improve motivation in young people with schizophrenia. Schizophr Bull 2018;44:1010-20.

34 Devi R, Carpenter C, Powell J, et al. Exploring the experience of using a web-based cardiac rehabilitation programme in a primary care angina population: a qualitative study. Int $J$ Ther Rehabil 2014;21:434-40.

35 Devi R, Powell J, Singh S. A web-based program improves physical activity outcomes in a primary care angina population: randomized controlled trial. J Med Internet Res 2014;16:e186.

36 Rawstorn JC, Gant N, Meads A, et al. Remotely delivered exercisebased cardiac rehabilitation: design and content development of a novel mHealth platform. JMIR Mhealth Uhealth 2016;4:e57.

37 Almeida RFSDE, Sousa TJ, Couto ANAS, et al. Development of weCope, a mobile APP for illness self-management in schizophrenia. Archives of Clinical Psychiatry 2019;46:1-4.

38 Mamykina L, Heitkemper EM, Smaldone AM, et al. Structured scaffolding for reflection and problem solving in diabetes selfmanagement: qualitative study of mobile diabetes detective. J Am Med Inform Assoc 2016;23:129-36.
39 Hans PK, Gray CS, Gill A, et al. The provider perspective: investigating the effect of the electronic patient-reported outcome (ePRO) mobile application and portal on primary care provider workflow. Prim Health Care Res Dev 2018;19:151-64.

40 Beatty AL, Magnusson SL, Fortney JC, et al. Va FitHeart, a mobile APP for cardiac rehabilitation: usability study. JMIR Hum Factors 2018;5:e3.

41 Steele Gray C, Gill A, Khan Al, et al. The electronic patient reported outcome tool: testing usability and feasibility of a mobile APP and portal to support care for patients with complex chronic disease and disability in primary care settings. JMIR Mhealth Uhealth 2016;4:e58.

42 Moy ML, Collins RJ, Martinez CH, et al. An internet-mediated pedometer-based program improves health-related quality-of-life domains and daily step counts in COPD: a randomized controlled trial. Chest 2015;148:128-37.

43 Moy ML, Martinez CH, Kadri R, et al. Long-term effects of an internet-mediated pedometer-based walking program for chronic obstructive pulmonary disease: randomized controlled trial. J Med Internet Res 2016;18:e215.

44 Wan ES, Kantorowski A, Homsy D, et al. Promoting physical activity in COPD: insights from a randomized trial of a web-based intervention and pedometer use. Respir Med 2017;130:102-10.

45 Demeyer H, Louvaris Z, Frei A, et al. Physical activity is increased by a 12-week semiautomated telecoaching programme in patients with COPD: a multicentre randomised controlled trial. Thorax 2017;72:415-23

46 Spruit MA, Pitta F, McAuley E, et al. Pulmonary rehabilitation and physical activity in patients with chronic obstructive pulmonary disease. Am J Respir Crit Care Med 2015;192:924-33.

47 Foster C, Fenlon D. Recovery and self-management support following primary cancer treatment. Br J Cancer 2011;105:S21-8.

48 Lorig KR, Ritter P, Stewart AL, et al. Chronic disease selfmanagement program: 2-year health status and health care utilization outcomes. Med Care 2001;39:1217-23.

49 Hartzler A, Venkatakrishnan A, Mohan S. Acceptability of a team-based mobile health (mHealth) application for lifestyle selfmanagement in individuals with chronic illnesses. Conference proceedings: Annual International Conference of the IEEE Engineering in Medicine and Biology Society IEEE Engineering in Medicine and Biology Society Annual Conference, 2016:3277-81.

50 Tomori K, Nagayama H, Saito Y, et al. Examination of a cut-off score to express the meaningful activity of people with dementia using iPad application (ADOC). Disabil Rehabil Assist Technol 2015;10:126-31.

51 Rosewilliam S, Roskell CA, Pandyan AD. A systematic review and synthesis of the quantitative and qualitative evidence behind patient-centred goal setting in stroke rehabilitation. Clin Rehabil 2011;25:501-14.

52 Scobbie L, Wyke S, Dixon D. Identifying and applying psychological theory to setting and achieving rehabilitation goals. Clin Rehabil 2009;23:321-33.

53 Tomori K, Uezu S, Kinjo S, et al. Utilization of the iPad application: aid for decision-making in occupation choice. Occup Ther Int 2012;19:88-97.

54 Bright FAS, Boland P, Rutherford SJ, et al. Implementing a clientcentred approach in rehabilitation: an autoethnography. Disabil Rehabil 2012;34:997-1004.

55 Gzil F, Lefeve C, Cammelli M, et al. Why is rehabilitation not yet fully person-centred and should it be more person-centred? Disabil Rehabil 2007;29:1616-24.

56 Becker S, Miron-Shatz T, Schumacher N, et al. mHealth 2.0: experiences, possibilities, and perspectives. JMIR Mhealth Uhealth 2014;2:e24.

57 Leach E, Cornwell P, Fleming J, et al. Patient centered goal-setting in a subacute rehabilitation setting. Disabil Rehabil 2010;32:159-72. 